\title{
Perishable Inventory Model Having Weibull Lifetime and Time Dependent Demand
}

\author{
Dr. R. John Mathew \\ Professor, Department Of Computer Science and Engineering, \\ Srinivasa Institute Of Engineering And Technology, A.P, India.
}

\begin{abstract}
In this paper we develop and analyse an inventory model for deteriorating items with Weibull rate of decay and time dependent demand. Using the differential equations, the instantaneous state of inventory at time 't', the amount of deterioration etc. are derived. With suitable cost considerations the total cost function and profit rate function are also obtained by maximizing the profit rate function, the optimal ordering and pricing policies of the model are derived. The sensitivity of the model with respect to the parameters is discussed through numerical illustration. It is observed that the deteriorating parameters have a tremendous influence on the optimal selling price and ordering quantity.
\end{abstract}

Keywords: Instantaneous rate of deterioration, Perishability, Profit rate function, the optimal ordering and pricing policies, Total cost function

\section{Introduction}

Much work has been reported regarding inventory models for deteriorating items in recent years. In many of the inventory systems the major consideration is regarding the pattern of demand and supply. Goyal and Giri [1] have reviewed inventory models for deteriorating items. They classified the literature by the self life characteristic of the inventory of goods. They also developed on the basis of demand variations and various other conditions or constraints. Various functional forms are considered for describing the demand pattern. Aggarwal and Goel [2] has developed an inventory model with the weibull rate of decay having selling price dependent demand. In reality, the demand rate of any product may vary with time or with price or with the instantaneous level of inventory. Inventory problems involving time dependent demand patterns have received the attention of several researchers in recent years. Giri, et al. [3], Mahata and Goswami [4], Manna, et al. [5]Mathew [6],[7], Ritchie [8], and Skouri, et al. [9] are among those who studied inventory models for deteriorating items having time dependent demand. It has been observed that for certain types of inventories, particularly consumer goods, heaps of stock will attract customers. Taking due account to this fact, Dye, et al. [10], Lee and Dye [11], Panda, et al. [12], Rao.K.S [13], and Venkata Subbaiah, et al. [14] and others have developed inventory models where demand rate is a function of on-hand inventory.

However, no serious attempt was made to develop inventory models for deteriorating items having weibull rate of decay and time dependent demand with finite rate of replenishment, which are very useful in many practical situations arising at oil and natural gas industry, photo chemical industries, chemical processes, etc. Hence, in this paper we develop and analysed an inventory model with the assumption that the lifetime of the commodity is random and follows a three-parameter weibull distribution having demand as a function of time with finite rate of replenishment. Using differential equations the instantaneous state of on hand inventory is obtained. With suitable cost considerations the total cost function is derived. The optimal ordering policies are also obtained. The sensitivity of the model is analysed though numerical illustration. This model includes some of the earlier models as particular cases for specific or limiting values of the parameters.

\section{Assumptions and Notations}

We adopt the following assumptions and notations for the models to be discussed.

\section{II.1 ASSUMPTIONS}

Assumption 1: The demand is known and is a function of unit selling price $\lambda(\mathrm{s})$.

Assumption 2: Replenishment rate is finite.

Assumption 3: Lead time zero

Assumption 4: A deteriorating item is lost

Assumption 5: The production rate is finite

Assumption 6: $\mathrm{T}$ is the fixed duration of a production cycle

Assumption 7: The lifetime of the commodity is random and follows a three parameter weibull distribution of the form $\mathrm{f}(\mathrm{t})=\alpha \beta(\mathrm{t}-\gamma)^{\beta-1} \mathrm{e}^{\alpha(\mathrm{t}-\mathrm{\gamma}) \beta}$ for $\mathrm{t}>\gamma$ where $\alpha, \beta, \gamma$ are parameters. 
Hence, the instantaneous rate of deterioration $h(t)$ is $h(t)=\alpha \beta(t-\gamma)^{\beta-1}$

\section{II.2 NOTATIONS}

$\begin{array}{ll}\text { Q } & : \text { The ordering quantity in one cycle of length T. } \\ \text { A } & : \text { The cost of placing an order. } \\ \text { C } & : \text { The cost price of one unit. } \\ \mathrm{h} & : \text { The inventory holding cost per unit per unit time. } \\ \mathrm{S} & : \text { The selling price of unit. } \\ \lambda(\mathrm{s}) & : \text { The demand rate, which is a function of unit selling price } \\ \mathrm{r} & : \text { The rate of demand. } \\ \mathrm{n} & : \text { The pattern index. }\end{array}$

\section{Inventory Model}

Consider an inventory system in which the amount of stock is zero at time $\mathrm{t}=0$. Replenishment starts at $\mathrm{t}=0$ and stops at $\mathrm{t}=\mathrm{t}_{1}$. The deterioration of the item starts after a certain fixed lifetime ' $\gamma$ '. Since the perishability starts after ' $\gamma$ ' the decrease in the inventory is due to demand during the period $(0, \gamma)$, demand and deterioration during the period $\left(\gamma, t_{1}\right.$. During $\left(t_{1}, T\right)$ the inventory level gradually decreases mainly to meet up demand and partly due to deterioration. By this process the stock reaches zero level at $\mathrm{t}=\mathrm{T}$. The cycle then repeats itself after time ' $\mathrm{T}$ '.

The schematic diagram representing the inventory system is shown in Fig.

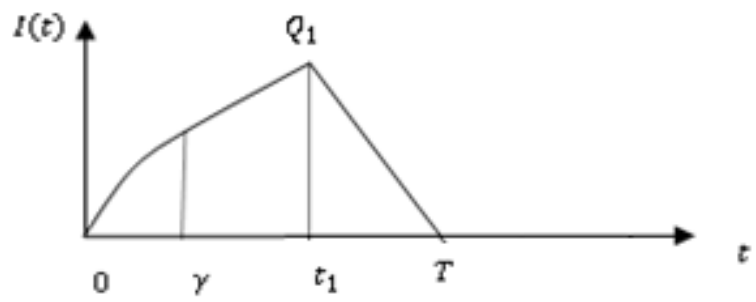

Schematic diagram representing the inventory level of the system.

Consider an inventory system in which the lifetime of the commodity is random and follows a three parameter weibull distribution with parameters $\alpha, \beta, \gamma$.

Hence the instantaneous rate of deterioration $\mathrm{h}(\mathrm{t})$ as

$$
\mathrm{H}(\mathrm{t})=\alpha, \beta(t-\gamma)^{\beta-1} \quad \text { for } \mathrm{t}>\gamma
$$

Along with all other assumptions made, assume that the demand rate is known and is a function time.

Let ' $Q$ ' be the ordering quantity in one cycle, ' $A$ ' be the cost of placing an order, and ' $C$ ' be the cost of one unit, ' $h$ ' be the inventory holding cost per unit per unit time. $\lambda(s, t)$ is assumed such that the total cost function is convex. In this model the stock decreases due to (i) the demand of items in the interval $(0, \gamma)$, (ii) the combination of deteriorating and demand of items in the interval $(\gamma, t)$, (iii) during $\left(t_{1}, T\right)$ the inventory level gradually decreases mainly to meet up demands and partly for deterioration, by this process the stock reaches zero level at $\mathrm{t}=\mathrm{T}$, The cycle then repeats itself after time $\mathrm{T}$.

Let $\mathrm{I}(\mathrm{t})$ be the inventory level of the system at time $\mathrm{t}(0 \leq \mathrm{t} \leq \mathrm{T})$. Then, the differential equations describing the instantaneous state of $\mathrm{I}(\mathrm{t})$ over the cycle of length $\mathrm{T}$ are,

$$
\begin{array}{llll}
\frac{d}{d t} I(t) & =\mathrm{k}-\frac{r t^{\frac{1}{n}-1}}{n T^{\frac{1}{n}}}, & & 0 \leq \mathrm{t} \leq \gamma \\
\frac{d}{d t} I(t)+\mathrm{h}(\mathrm{t}) \mathrm{I}(\mathrm{t})=\mathrm{k}-\frac{r t^{\frac{1}{n}-1}}{n T^{\frac{1}{n}}}, & \gamma \leq \mathrm{t} \leq t_{1} \\
\frac{d}{d t} I(t)+\mathrm{h}(\mathrm{t}) \mathrm{I}(\mathrm{t})=-\frac{r t^{\frac{1}{n}-1}}{n T^{\frac{1}{n}}}, & t_{1} \leq \mathrm{t} \leq \mathrm{T}
\end{array}
$$

with the initial conditions I $(0)=0,, \mathrm{I}(\mathrm{T})=0$.

Solving the above differential equations (3.1) to (3.3), the on hand inventory at time $t$ can be obtained as,

$$
\mathrm{I}(\mathrm{t})=\mathrm{kt}-\frac{r t^{\frac{1}{n}}}{T^{\frac{1}{n}}}, 0 \leq \mathrm{t} \leq \gamma
$$


$\mathrm{I}(\mathrm{t})=e^{-\alpha(t-\gamma)^{\beta}}\left\{\int_{\gamma}^{t}\left[\mathrm{k}-\frac{r u^{\frac{1}{n}-1}}{n T^{\frac{1}{n}}}\right] e^{\left.-\alpha(u-\gamma)^{\beta} d u+\mathrm{k} \gamma-\frac{r \gamma^{\frac{1}{n}}}{T^{\frac{1}{n}}}\right\}}, \gamma \leq \mathrm{t} \leq t_{1}\right.$
$\mathrm{I}(\mathrm{t})=e^{-\alpha(t-\gamma)^{\beta}} \frac{r}{n T^{\frac{1}{n}}}\left[\int_{t_{1}}^{t^{2}} t^{\frac{1}{n}-1} e^{\propto(t-\gamma)^{\beta}} d u-\int_{t_{1}}^{t} u^{\frac{1}{n}-1} e^{\propto(u-\gamma)^{\beta}} d u\right], t_{1} \leq t \leq T$

The stock loss due to deterioration in the interval $(0, \mathrm{~T})$ is

$\mathrm{I}(\mathrm{t})=\mathrm{k} t_{1}-\frac{r t^{\frac{1}{n}}}{n T^{\frac{1}{n}}} t_{2}$

The ordering quantity in a cycle of length $\mathrm{T}$ is obtained as

$\mathrm{Q}=\mathrm{Kt}_{1}$

Let $\mathrm{K}\left(\mathrm{t}_{1}, \mathrm{~T}\right.$.) be the expected total cost per unit time since the total cost is the sum of the set up cost, cost of the units, the inventory holding cost. $\mathrm{K}\left(\mathrm{t}_{1}, \mathrm{~T},\right)$ is obtained as

$$
\begin{aligned}
\mathrm{K}\left(\mathrm{t}_{1}, \mathrm{~T}\right)= & \frac{A}{T}+\frac{c Q}{T}+\frac{h}{T}\left[\int_{0}^{\gamma} h(t) d t+\int_{\lambda}^{t_{1}} h(t) d t+\int_{t_{1}}^{t_{2}} h(t) d t\right]+\frac{\pi}{T} \int_{t_{2}}^{t_{3}} I(t) d t \\
\mathrm{k}\left(\mathrm{t}_{1}, \mathrm{~T}\right)= & \frac{A}{T}+\frac{c}{T}\left[k t_{1}\right]+\frac{h}{T} \int_{o}^{r}\left[k t-\frac{r^{t^{\frac{1}{n}}}}{T^{\frac{1}{n}}}\right] d t \\
& +\frac{\boldsymbol{h}}{\boldsymbol{T}}\left\{\int_{r}^{t_{1}} e^{-a(t-r)^{\beta}}\left[\int_{r}^{t}\left(k-\frac{r^{\cdot t^{\frac{1}{n}-1}}}{n \cdot T^{\frac{1}{n}}}\right) e^{\propto(t-r)^{\beta}} d t+k \gamma-\frac{r \cdot \gamma^{\frac{1}{n}}}{T^{\frac{1}{n}}}\right] d t\right\} \\
& +\frac{h r}{n T^{\frac{1}{n}+1}}\left\{\int_{t_{1}}^{T} e^{-\propto(t-r)^{\beta}}\left[\int_{t_{1}}^{T} t^{\frac{1}{n}-1} e^{\propto(t-r)^{\beta}} d t-\int_{t_{1}}^{t} u^{\frac{1}{n}-1} e^{\propto(u-r)^{\beta}} d t\right] d t\right\}
\end{aligned}
$$

Using tailors series expansion for the exponential function for small values of $\alpha(t-r)^{\beta}$ and ignoring higher order terms and on simplification, we have

$$
\begin{aligned}
& k\left(t_{1}, T\right)=\frac{A}{T}+\frac{c k t_{1}}{T}+\frac{h}{T} k\left[\frac{T^{2}}{2}+\frac{2 \propto}{(\beta+1)(\beta+2)}\left(t_{1}-\gamma\right)^{\beta+2}-\frac{\propto t_{1}\left(t_{1}-\gamma\right)^{\beta+1}}{\beta+1}\right] \\
& -\frac{h n r}{T^{\frac{1}{n}+1}}\left\{\frac{\propto}{n \beta+1}\left[\frac{-\gamma^{\frac{1}{n}+\beta+1}}{\frac{1}{n}+\beta+1}-\gamma^{\frac{1}{n}+\beta}\left(t_{1}-\gamma\right)\right]-\frac{\propto \beta \gamma}{n \beta-n+1}\left[\frac{-\gamma^{\frac{1}{n}+\beta}}{\frac{1}{n}+\beta}-\gamma^{\frac{1}{n}+\beta-1}\left(t_{1}-\gamma\right)\right]\right. \\
& \left.+\propto \frac{\gamma^{\frac{1}{n}+\beta+1}}{\frac{1}{n}+\beta+1}-\left[\frac{\propto \beta \gamma^{\frac{1}{n}+\beta+1}}{\frac{1}{n}+\beta}\right]\right\}+\frac{h r}{T^{\frac{1}{n}+1}}\left\{T^{\frac{1}{n}+1}-t_{1} T^{\frac{1}{n}}-\frac{T^{\frac{1}{n}+1}}{\frac{1}{n}+1}+\frac{\propto}{n \beta+1}\left[T^{\frac{1}{n}+\beta}\left(T-t_{1}\right)-\frac{T^{\frac{1}{n}+\beta+1}}{\frac{1}{n}+\beta+1}\right]\right. \\
& \left.-\frac{\propto \beta \gamma}{n \beta-n+1}\left[T^{\frac{1}{n}+\beta-1}\left(T-t_{1}\right)-\frac{T^{\frac{1}{n}+\beta}}{\frac{1}{n}+\beta}\right]-\frac{\propto T^{\frac{1}{n}}}{\beta+1}\left[(T-\gamma)^{\beta+1}-\left(t_{1}-r\right)^{\beta+1}\right]+\propto \frac{T^{\frac{1}{n}+\beta+1}}{\frac{1}{n}+\beta+1} \propto \beta \gamma \frac{T^{\frac{1}{n}+\beta}}{\frac{1}{n}+\beta}\right\}
\end{aligned}
$$

\section{Optimal Policies Of The Model}

For obtaining the optimal polices of the perishable inventory model having deterministic demand as power function of time, the expected total cost per unit time $\mathrm{k}\left(\mathrm{t}_{1}, \mathrm{~T}\right)$ is obtained.To find the optimal values of $\mathrm{t}_{1}$, 
$T$ equate the first order partial derivatives of $K\left(t_{1}, T\right)$ with respect to $t_{1}, T$ to zero.By differentiating $K\left(t_{1}, T\right)$ with respect to $t_{1}$ and equating to zero, we get

$$
\begin{aligned}
& \frac{c k}{T}+\frac{h k}{T}\left[t_{1}+\frac{\alpha}{\beta+1}-\left(t_{1}-\gamma\right)^{\beta+1}-\propto t_{1}\left(t_{1}-\gamma\right)^{\beta}\right]-\frac{h r}{T^{\frac{1}{n}+1}}\left[\frac{-\alpha \gamma^{\frac{1}{n}+\beta}}{n \beta+1}+\frac{\alpha \beta \gamma^{\frac{1}{n}+\beta}}{n \beta-n+1}\right] \\
& +\frac{h r}{T^{\frac{1}{n}+1}}\left[-T^{\frac{1}{n}}-\frac{\alpha}{n \beta+1} T^{\frac{1}{n}+\beta}+\frac{\alpha \beta \gamma}{n \beta-n+1} T^{\frac{1}{n}-\beta+1}+\alpha T^{\frac{1}{n}}\left(t_{1}-\gamma\right)^{\beta}\right]=0
\end{aligned}
$$

By differentiating $\mathrm{K}\left(\mathrm{t}_{1}, \mathrm{~T}\right)$ With respect to $\mathrm{T}$ and equating to zero, we get

$$
\begin{aligned}
& -A-c k t_{1}-h k\left[\frac{t_{1}^{2}}{2}+\frac{2 \alpha\left(t_{1}-\gamma\right)^{(\beta+2)}}{(\beta+1)(\beta+2)}-\frac{\alpha t_{1}\left(t_{1}-\gamma\right)^{(\beta+1)}}{\beta+1}\right]+\frac{h r(n+1)}{T^{\frac{1}{n}}}\left\{\frac{\alpha}{n \beta+1}\left[-\frac{\gamma^{\frac{1}{n}+\beta+1}}{\frac{1}{n}+\beta+1}-\gamma^{\frac{1}{n}+\beta}\left(t_{1}-\gamma\right)^{(\beta+1)}\right]\right. \\
& \left.+\frac{\alpha \beta \gamma}{n \beta-n+1}\left[\frac{\gamma^{\frac{1}{n}+\beta}}{\frac{1}{n}+\beta}+\gamma^{\frac{1}{n}+\beta-1}\left(t_{1}-\gamma\right)\right]+\frac{\alpha \gamma^{\frac{1}{n}+\beta+1}}{\frac{1}{n}+\beta+1}-\frac{\alpha \beta \gamma^{\frac{1}{n}+\beta+1}}{\frac{1}{n}+\beta}\right\}+T^{2} h r\left\{\frac{t_{1}}{T^{2}}+\frac{\alpha}{n \beta+1}\left[\beta T^{\beta-1}-(\beta-1) t_{1} T^{\beta-2}-\frac{\beta T^{\beta-1}}{\frac{1}{n}+\beta+1}\right]\right. \\
& \frac{\alpha \beta \gamma}{n \beta-n+1}\left[(\beta-1) t_{1} T^{\beta-2}-(\beta-2) t_{1} T^{\beta-3}-\frac{(\beta-1) T^{\beta-2}}{\frac{1}{n}+\beta}\right]-\frac{\alpha}{T^{2}(\beta+1)}\left[(\beta+1) T(T-\beta)^{\beta}-(T-\gamma)^{\beta+1}+\left(t_{1}-\gamma\right)^{\beta+1}\right] \\
& \left.+\frac{\alpha \beta T^{\beta-1}}{\frac{1}{n}+\beta+1}-\frac{\alpha \beta \gamma(\beta-1) T^{\beta-2}}{\frac{1}{n}+\beta}\right\}=0
\end{aligned}
$$

Solving equations (4.1) and (4.2) numerically for given values of $\mathrm{T}, \alpha, \beta, \gamma, r, n, A, c$ and $h$ one can obtain the optimal values $\mathrm{t}_{1}{ }^{*}$ of $\mathrm{t}_{1}, \mathrm{~T}^{*}$ of $\mathrm{T}$ and also obtain the optimal expected total cost per unit time. Even though $\mathrm{T}$ is fixed the expected total cost is a function of $\mathrm{T}$. So the expected cost function is effected by the various values of $\mathrm{T}$. Hence the optimal cycle length can also be obtained by using search methods after substituting the optimal value of $\mathrm{t}_{1}{ }^{*} \mathrm{~T}^{*}$ and $\mathrm{Q}^{*}$ as initial values.

\section{Numberical Illustration}

For various values of $\alpha, \beta, \gamma$ and $\mathrm{T}$ the optimal values of $\mathrm{t}_{1} *, \mathrm{~T}^{*}, \mathrm{~s}^{*}$ and $\mathrm{Q}^{*}$ are computed by solving the equations (4.1),(4.2) and (4.3) and presented on table 1 . From Table 1 , it is observed that the decision variables of the system namely the unit selling price, the ordering quantity and the time of stopping the production are influenced by the production rate ' $\mathrm{K}$ '. As ' $\mathrm{K}$ ' increases the unit selling price is decreasing, and the optimal time period of production is increasing for fixed values of the parameters. That is if the capacity utilization of the production process is maximum then the selling price and the ordering quantity are optimal.

Table -1

Optimum values of production time, , total cost and ordering quantity

\begin{tabular}{llllllll|l|l|l|l|l}
$\mathrm{c}$ & $\mathrm{h}$ & $\mathrm{r}$ & $\mathrm{n}$ & $\mathrm{k}$ & $\alpha$ & $\beta$ & $\gamma$ & $\mathrm{A}$ & $\mathrm{t}_{1}$ & $\mathrm{~T}$ & $\mathrm{~K}$ & $\mathrm{Q}$ \\
4 & 0.4 & 3 & 2 & 5 & 3 & 2 & 0 & 50 & 1.129 & 5.882 & 14.154 & 8.936 \\
5 & 0.4 & 3 & 2 & 5 & 3 & 2 & 0 & 50 & 1.319 & 6.617 & 16.154 & 90210 \\
6 & 0.4 & 3 & 2 & 5 & 3 & 2 & 0 & 50 & 1.466 & 6.617 & 18.154 & 9.314 \\
4 & 0.4 & 3 & 2 & 5 & 3 & 2 & 0 & 50 & 1.129 & 5.882 & 14.154 & 8.936 \\
4 & 0.5 & 3 & 2 & 5 & 3 & 2 & 0 & 50 & 1.104 & 5.362 & 14.470 & 8.945 \\
4 & 0.6 & 3 & 2 & 5 & 3 & 2 & 0 & 50 & 1.082 & 4.973 & 14.786 & 8.994 \\
4 & 0.4 & 2 & 2 & 5 & 3 & 2 & 0 & 50 & 1.310 & 6.493 & 13.369 & 8.576 \\
4 & 0.4 & 3 & 2 & 5 & 3 & 2 & 0 & 50 & 1.129 & 5.882 & 14.154 & 8.936 \\
4 & 0.4 & 4 & 2 & 5 & 3 & 2 & 0 & 50 & 0.869 & 5.403 & 14.939 & 9.102
\end{tabular}




\begin{tabular}{|c|c|c|c|c|c|c|c|c|c|c|c|c|}
\hline 4 & 0.4 & 3 & 1 & 5 & 3 & 2 & 0 & 50 & 1.399 & 4.973 & 14.860 & 8.524 \\
\hline 4 & 0.4 & 3 & 2 & 5 & 3 & 2 & 0 & 50 & 1.290 & 5.552 & 14.154 & 8.936 \\
\hline 4 & 0.4 & 3 & 3 & 5 & 3 & 2 & 0 & 50 & 0.927 & 6.718 & 13.729 & 9.012 \\
\hline 4 & 0.4 & 3 & 2 & 5 & 3 & 2 & 0 & 50 & 1.349 & 5.598 & 14.191 & 8.714 \\
\hline 4 & 0.4 & 3 & 2 & 5 & 3 & 2 & 0 & 50 & 1.129 & 5.882 & 14.154 & 8.976 \\
\hline 4 & 0.4 & 3 & 2 & 5 & 3 & 2 & 0 & 50 & 0.787 & 6.041 & 14.117 & 9.102 \\
\hline 4 & 0.4 & 3 & 2 & 5 & 3 & 2 & 0 & 50 & 1.129 & 5.882 & 14.154 & 8.936 \\
\hline 4 & 0.4 & 3 & 2 & 4 & 3 & 2 & 0 & 50 & 0.945 & 5.521 & 12.794 & 8.726 \\
\hline 4 & 0.4 & 3 & 2 & 5 & 3 & 2 & 0 & 50 & 1.129 & 5.882 & 14.154 & 8.936 \\
\hline 4 & 0.4 & 3 & 2 & 6 & 3 & 2 & 0 & 50 & 1.226 & 6.155 & 15.514 & 9.102 \\
\hline 4 & 0.4 & 3 & 2 & 5 & 3 & 2 & 0 & 50 & 1.023 & 5.722 & 15.655 & 8.412 \\
\hline 4 & 0.4 & 3 & 2 & 5 & 3 & 2 & 0 & 50 & 1.082 & 5.809 & 14.813 & 8.216 \\
\hline 4 & 0.4 & 3 & 2 & 5 & 3 & 2 & 0 & 50 & 1.129 & 5.882 & 14.154 & 8.936 \\
\hline 4 & 0.4 & 3 & 2 & 5 & 2 & 2 & 0 & 50 & 1.217 & 6.988 & 13.866 & 8.512 \\
\hline 4 & 0.4 & 3 & 2 & 5 & 3 & 2 & 0 & 50 & 1.129 & 5.882 & 14.154 & 8.936 \\
\hline 4 & 0.4 & 3 & 2 & 5 & 4 & 2 & 0 & 50 & 1.063 & 5.205 & 14.442 & 9.124 \\
\hline 4 & 0.4 & 3 & 2 & 5 & 3 & 2.0 & 0 & 50 & 1.129 & 5.882 & 14.154 & 8.936 \\
\hline 4 & 0.4 & 3 & 2 & 5 & 3 & 2.5 & 0 & 50 & 1.278 & 4.391 & 17.012 & 9.126 \\
\hline 4 & 0.4 & 3 & 2 & 5 & 3 & 2.0 & 0 & 50 & 1.293 & 3.567 & 24.432 & 9.234 \\
\hline 4 & 0.4 & 3 & 2 & 5 & 3 & 2 & 0 & 50 & 1.129 & 2.736 & 14.154 & 8.936 \\
\hline 4 & 0.4 & 3 & 2 & 5 & 3 & 2 & 0.1 & 50 & 1.860 & 3.905 & 14.213 & 9.128 \\
\hline 4 & 0.4 & 3 & 2 & 5 & 3 & 2 & 0.2 & 50 & 1.984 & 3.154 & 14.245 & 9.224 \\
\hline 4 & 0.4 & 3 & 2 & 5 & 3 & 2 & 0 & 50 & & & 14.154 & 8.936 \\
\hline 4 & 0.4 & 3 & 2 & 5 & 3 & 2 & 0 & 75 & & & 16.745 & 12.428 \\
\hline 4 & 0.4 & 3 & 2 & 5 & 3 & 2 & 0 & 100 & & & 19.245 & 16.156 \\
\hline
\end{tabular}

From Table 1 it is observed that the optimal ordering quantity, the expected total cost per unit time is much influenced by the parameters, various costs and cycle length. It is observed that as cycle length increases, the optimal ordering quantity is increasing when the other parameters and cost are fixed. The optimal ordering quantity is also influenced by the mean lifetime of the commodity. The optimal ordering quantity is very sensitive to the penalty cost, when other parameters are fixed. When the penalty cost is increasing, the optimal ordering quantity is increasing. However if the increase in the penalty cost is in proportion to the increase in the cost per unit, then the optimal ordering quantity is an increasing function to the increase in the cost. If the cost per unit is much higher than the penalty cost, then the optimal ordering quantity is a decreasing function of the cost per unit. The optimal ordering quantity is a decreasing function of the holding cost. However even if the holding cost is much less compared to the penalty cost then optimal ordering quantity is decreasing function of the holding cost. So by properly choosing the penalty cost one can have the optimal inventory management. It is also observed that the parameters of the model influence the mean lifetime of the commodity and hence the rate of deterioration, which results in the change of the optimal ordering quantity. The optimal ordering quantity is a decreasing function of the location parameter, $\gamma$ increases, As a result of the decrease in the optimal ordering quantity, the total expected cost per unit time is decreasing. Since the location and shape parameters of the lifetime of the commodity have a significant influence on the rate of deterioration, there is a need to analyze the influence of these two parameters on the optimal values of their inventory models with respect to their interrelationship Even though $\mathrm{T}$, the cycle length is considered to be a known value in this inventory model, it is also interesting to note that the cycle length has a vital influence on the optimal ordering quantity and expected total cost per unit time. For deriving the optimal ordering quantity one has to properly estimate the parameters involved in the lifetime of the commodity.

\section{Sensitivity Analysis}

In order to study how the parameters affect the optimal solution sensitivity analysis is carried out taking the values $\mathrm{c}=2, \mathrm{~h}=0.2, \mathrm{r}=5, n=2, \mathrm{k}=2, \alpha=2 \beta=2.2, \gamma=0$, and $\mathrm{A}=5$ in appropriate units. Sensitivity analysis is performed by decreasing and increasing these parameter values. First changing the value of one parameter at a time while keeping all the rest at their true values and then changing the values of all the parameters simultaneously. The result of this analysis is given in Table 1. From Table 1 we observe that the production 
downtime $t_{1} *$ is moderately sensitive to $\alpha$ and $p$ and slightly sensitive to the changes in other parameter values. The optimal production quantity $Q *$ is highly sensitive to the deterioration distribution parameter $\alpha$ and less sensitive to others.

\section{Conclusions}

Inventory models play a dominant role in manufacturing and production industries like cement, food processing, petrochemical, and pharmaceutical and paint manufacturing units. In this paper, an inventory model for deteriorating items with constant rate of replenishment, time and selling price dependent demand and Weibull decay has been developed and analyzed in the light of various parameters and costs and with the objective of maximizing the total system profit. The model was illustrated with numerical examples and sensitivity analysis of the model with respect to costs and parameters was also carried out. This model also includes the exponential decay model as a particular case for specific values of the parameters. The proposed model can further be enriched by incorporating salvage of deteriorated units, inflation, quantity discount, and trade credits etc. It can also be extended to a multi-commodity model with constraints on budget, shelf space, etc., These models may also be formulated in fuzzy environments.

\section{References}

[1] Goyal, S.K.,Giri, B.C (2001) Inview recent trends in modeling of deteriorating inventory, EJOR , Vol. 134,1 -16.

[2] Aggarwal, S.P., Goel, V.P (1984) Order Level inventory system with demand pattern for deteriorating items, Econ. Comp. Econ. Cybernet, Stud. Res., Vol. 3,57- 69.

[3]. Giri, B.C., Goswami, A. and Chaudhuri, K. S. (1996). An EOQ model for deteriorating items with time varying demand and costs Journal of the Operational Research Society, Vol.47, 1398-1405.

[4]. Mahata G. C. and Goswami A. (2009a) 'Fuzzy EOQ Models for Deteriorating Items with Stock Dependent Demand \& Non-Linear Holding Costs', International Journal of Applied Mathematics and Computer Sciences 5;2, 94-98.

[5]. Manna, S.K., Chaudhuri, K.S. and Chiang, C. (2007) 'Replenishment policy for EOQ models with time-dependent quadratic demand and shortages', International journal of Operational Research, Vol. 2, No.3 pp. $321-337$

[6] Mathew, R.J., Narayana, J.L. (2007) Perishable inventory model with finite rate of replenishment having weibull lifetimeand price dependent demand Assam Statistical review (2007), Vol. 21, 91-102.

[7] Mathew, R.J(2013) Perishable inventory model with finite rate of replenishment having weibull lifetime and time dependent demand .Accepted by International journal of mathematical archive,IJMR; $4-228$

[8]. Ritchie, E. (1984). The EOQ for linear increasing demand, A simple optimum solution. Journal of the Operational Research Society, Vol.35, 949-952.

[9]. Skouri, K., Konstantaras, I., Papachristos, S., Ganas, I., (2009) 'Inventory models with ramp type demand rate, partial backlogging and Weibull deterioration rate', European Journal of Operational Research, Vol. 192 (1), 79-92.

[10]. Dye C.Y., Hsieh T.P., and Ouyang L.Y. (2007) 'Determining optimal selling price and lot size with a varying rate of deterioration and exponential partial backlogging', European Journal of Operational Research, Vol. 181(2), 668-678

[11]. Yu-Ping Lee Chung-Yuan Dye (2012). An inventory model for deteriorating items under stock-dependent demand and controllable deterioration rate. Computers \& Industrial Engineering (63), 474-482

[12]. Panda S. Saha S. and Basu M. (2009). An EOQ model for perishable products with discounted selling price and stock dependent demand. Central European Journal of Operations Research, 17:31-53

[13]. Srinivasa Rao K., Uma Maheswara Rao S. V., Venkata Subbaiah K. (2011). Production inventory models for deteriorating items with production quantity dependent demand and Weibull decay. International Journal of Operational Research, 11 (1), 31 - 53.

[14]. Venkata Subbaiah K., Srinivasa Rao K. and Satyanarayana B. (2004) 'Inventory models for perishable items having demand rate dependent on stock level', Opsearch, Vol. 41, No 4, 222-235. 